\title{
HAEMANGIOMA OF THE ORBIT, REMOVED BY OPERATION
}

\author{
BY \\ Major T. CRAWFORD, R.A.M.C. \\ SPECIALIST IN PATHOLOGY \\ Major E. F. KING, R.A.M.C. \\ SPECIALIST IN OPHTHALMOLOGY \\ and \\ Major H. W. RODGERS, R.A.M.C. \\ SPECIALIST IN SURGERY
}

Private B, aged 30, a regular soldier, reported sick in Hong Kong in February, 1940.

The man gave a history that eighteen months previously the right eye became inflamed and felt " tense" and that he experienced a sense of numbness in the right temple; these symptoms passed off in a few days. For the last six months he had experienced intermittent attacks of neuralgic pain behind and over the eye, lasting for several hours; these were increasing in severity and leading to some loss of sleep. At no time had he noticed any head noises.

He was seen by an ophthalmic specialist who reported a mild degree of right proptosis. General and special examinations failed to reveal any other abnormality. He was kept under observation and as the proptosis slowly progressed he was returned to the U.nited Kingdom.

When seen in this country in January 1942 he showed wellmarked right proptosis, directly forwards, unassociated with any retraction or lagging of the upper lid. No pulsation or bruit was detected. The movements of the globe were surprisingly full though there was some limitation of its peripheral excursions, particularly upwards and inwards. No sensory changes were detected in the cornea or in the skin adjacent to the eye. Deep palpation of the orbit failed to reveal any mass. The pupil reacted well to light and on accommodation. The optic disc and surrounding fundus were normal. There was slight, though definite, nasal contraction of the peripheral visual field (see Chart-Fig. 1). The blind spot was normal and no central scotoma was detected.

Vision $=\frac{6}{18} \mathrm{c}+1.00$ sphere 1.00 cylinder $180^{\circ}=\frac{6}{9}$ and J.1. The man had never worn glasses and was emphatic that the vision in the eye had deteriorated in the last two years and it seemed possible that the hypermetropia and astigmatism were, in part, artificially produced by pressure on the posterior pole of the eve. 


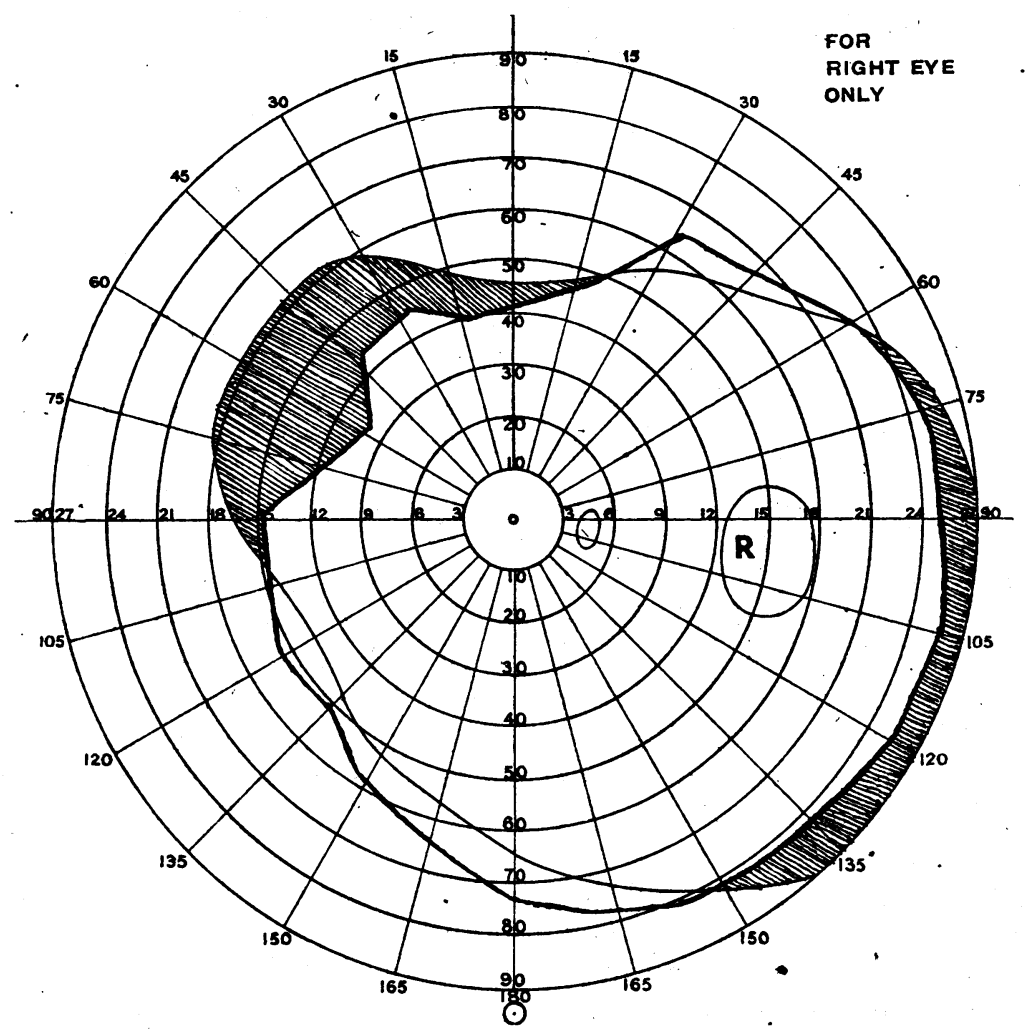

FIG. 1.

The left eye was essentially normal, and the vision $\frac{+0.50 \mathrm{sph} .}{\mathrm{c}+0.50 / 180^{\circ}}=\frac{6}{5}$.

$\mathrm{X}$-rays showed both optic canals of equal size and there were no abnormal shadows in the orbits or sinuses.

Complete general and special investigations were carried out, particularly in regard to a primary malignant tumour elsewhere in the body or any changes in the paranasal sinuses. All were negative.

- The diagnosis of a slowly growing tumour of the orbit within the cone of muscles was made and surgical exploration advised.

\section{Operation}

The usual approach to the retro-ocular contents by reflection of the lateral orbital wall was used: The alternative transcranial exposure, by-removing the posterior two-thirds of the orbital roof, 
was not undertaken in this case as it was thought improbable that the orbital tumour had any intracranial connections.

Several incisions in the bone have been recommended. Of these the widest exposure, recommended by Kocher, is given by dividing the bone along the following lines (see Fig. 2): (A) From the zygomatic process of the frontal bone to the middle of the inferior orbital fissure. (13) From the lower and outer angle of the orbit,

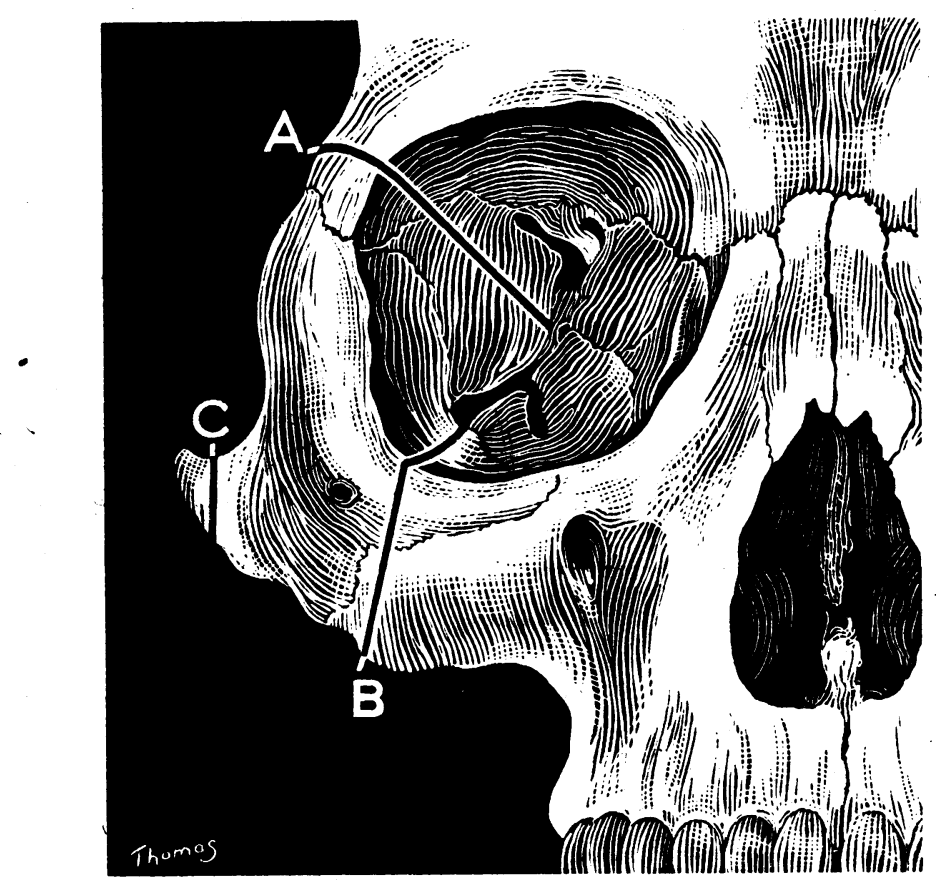

FIG. 2.

backwards and inwards to the anterior extremity of the inferior orbital fissure. (C) Across the zygomatico-temporal suture.

The preliminary skin incision (see Fig. 3) was carried deeply to the zygomatic process of the frontal bone. The orbital contents were displaced medially by the cautious separation of the thin and ill-defined orbital periosteum until the orbital fissure was reached. In this a malleable retractor was placed to protect the orbital contents and, keeping in close contact with the calvarium, the first $(\mathrm{A})$ of the bone incisions described above was begun with a small saw and completed with a chisel. Before the next cut (B) was made a blunt hook was placed in the anterior end of the inferior orbital fissure to act as a guide, and the incision was made sufficiently lateral to avoid the maxillary air sinus. Subsequently, the 


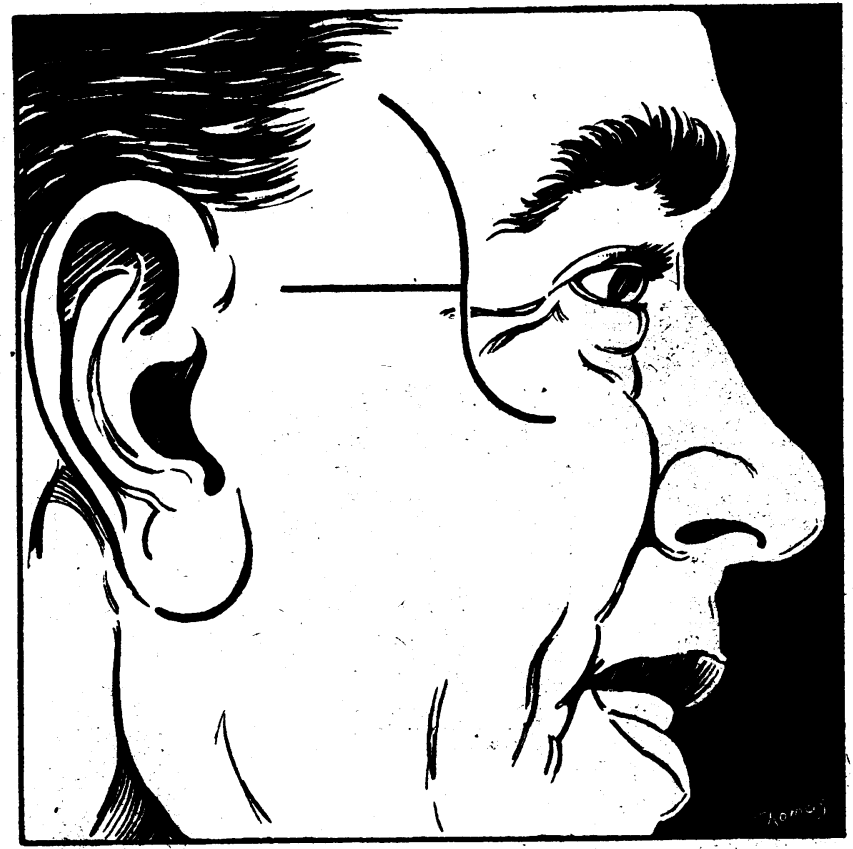

FIG.-3.

zygomatic arch having been divided, the zygoma with its attached soft and bone tissues was readily displaced backwards giving an excellent exposure for the next important stage of the operation.

It was not necessary to divide the lateral rectus which was retracted upwards by a hook. Blunt dissection of the orbital fat revealed the tumour, of dark red colour and irregular in shape. It was lying within the cone of muscles in close apposition, but not attached, to the optic nerve. The tumour was readily and completely removed by separation with the forefinger.

This operation destroyed both branches of the zygomatic nerve. A more conservative approach might have preserved the zygomatico-facial branch at the expense of a more cramped field of operation with its attendant risk of irreparable damage to the delicate retro-ocular structures.

The, only difficulty experienced in closing the wound was the accurate resetting of the orbital margin; but good alignment was finally attained by a few strong catgut sutures.

There were two notable features of this wide and apparently mutilating exposure, namely, the absence of haemorrhage, and the small degree of permanent disfigurement produced. 
Convalescence following the operation was uneventful apart froni marked oedema of the upper lid which, on examination six weeks later, had subsided considerably.

The proptosis was entirely relieved and movements of the globe were full apart from some weakness of the external rectus muscle. The pupil, which previous to the operation had been normal, was now semi-dilated and reacted sluggishly to light and on accommodation. There was also marked cycloplegia.

The vision was $\frac{6}{24} \mathrm{c}+2 \cdot 50 \mathrm{sph} .=\frac{6}{6}$, and it was necessary to add $\mathrm{a}+2 \cdot 0 \mathrm{sph}$. to obtain $\mathrm{J}: 1$.

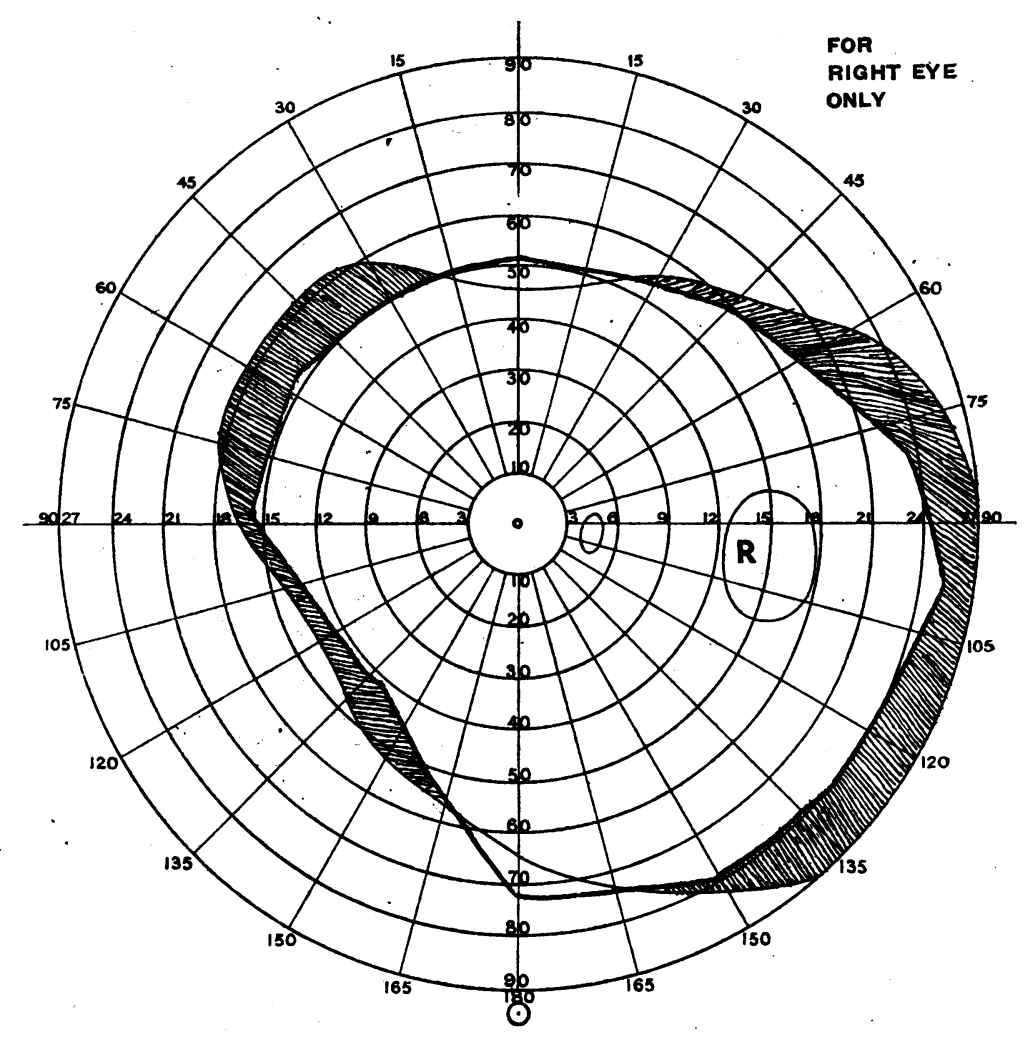

FIG. 4.

It seems that this post-operative increase in hypermetropia was due to the cycloplegia which made manifest the whole of the latent hypermetropia.

The fundus was still normal. The visual field was now full there being no trace of the nasal contraction previously noted (see Chart-Fig. 4). 


\section{Pathological Report (See Figs. 5 and 6)}

The tumour is roughly egg-shaped and measures $2-3 \mathrm{~cm}$. in length by $1.5 \mathrm{~cm}$. in width. The cut surface has a fleshy appearance with areas suggesting blood clot.

Microscopic examination shows the tumour to be composed largely of cavernous blood-containing spaces lined by welldeveloped vascular endothelium,' separated by fibrous tissue of

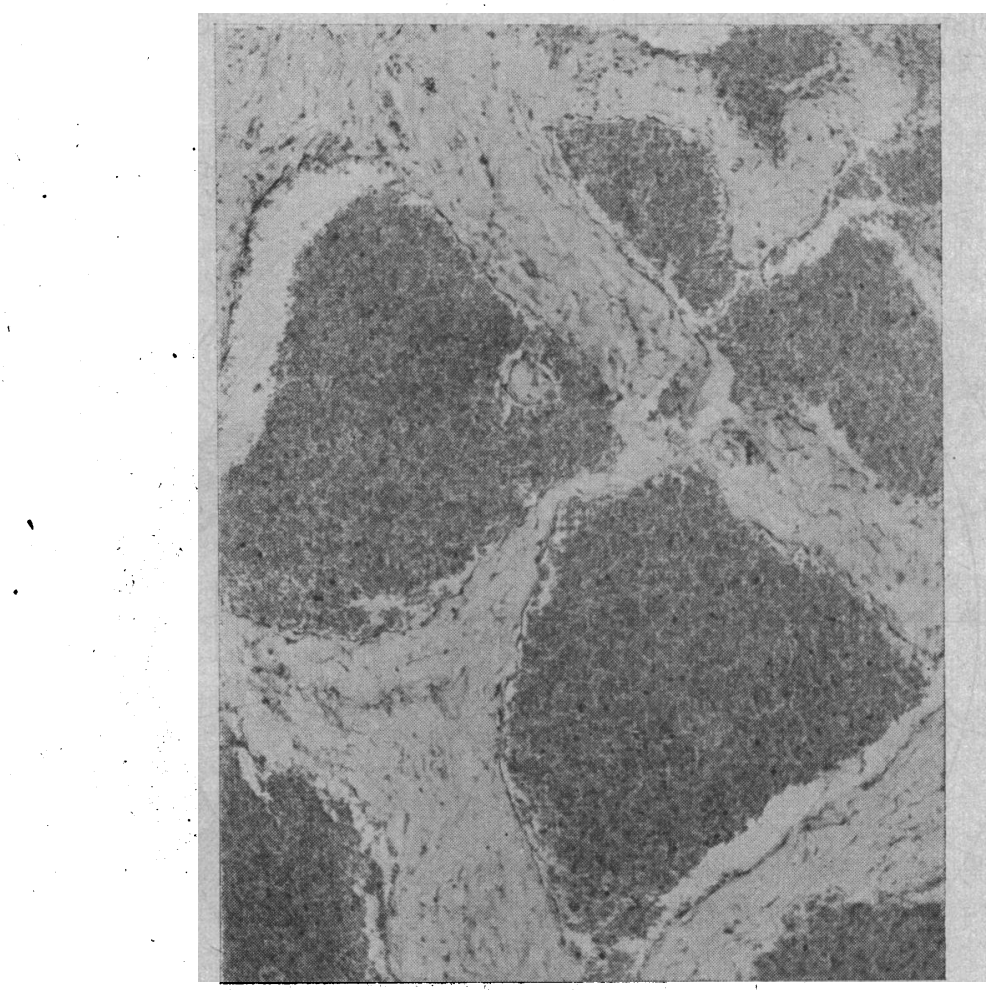

FIG. 5.

Low power view (magnification 40 times) showing general structure of the tumour. Large blood-containing spaces separated by bands of hyaline connective tissue.

varying density. In addition to the cavernous spaces a few small arterioles with muscular walls are present. While the bulk of the tumour is formed of these mature vascular elements there are, in addition, areas in which solid cords of endothelial cells separate primitive capillary channels. These areas show numerous mitotic figures and clearly constitute the growing foci of the tumour. 
Numerous blood cells have escaped into the interstitial tissue between the cavernous spaces, and haemosiderin granules are present, in large numbers, within the cytoplasm of endothelial cells and macrophages. At the edges of the tumour the vascular elements

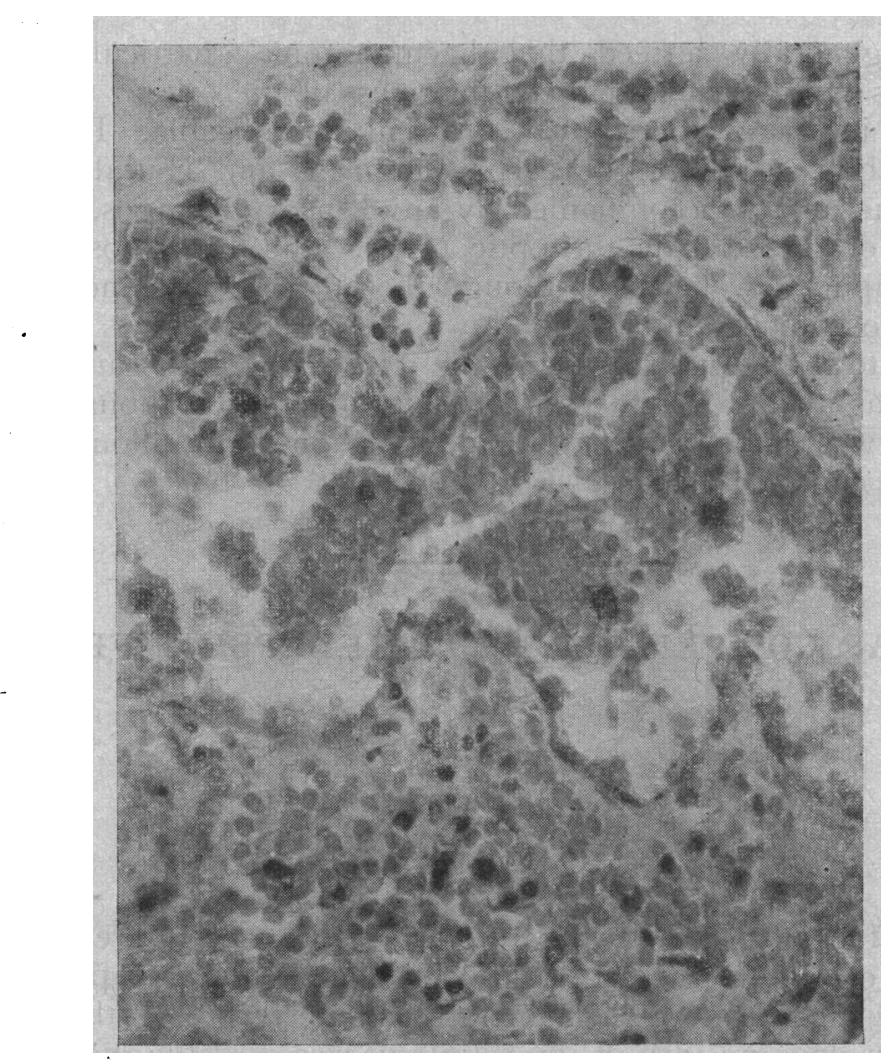

FIG. 6.

High power view (magnification 250 times) showing a blood-containing space, lined by a single layer of flattened endothelial cells, some of which contain haemosiderin granules. Many blood corpuscles have escaped from the blood vessel, and can be seen lying free amongst the supporting connective tissue.

fade gradually into the " capsule" formed of a loose fibrous and adipose tissue.

The tumour is clearly a mixed capillary and cavernous haemangioma arising, presumably, from the blood vessels lying amongst the orbital fatty tissues. The possibility of its origin from the sheath of Schwalbe has been considered, but there is no histological evidence to support this. 
While the tumour is essentially simple in nature, the microscopic appearances suggest the possibility of rapid growth or rapid recurrence following incomplete removal.

\title{
Conclusion
}

It is generally agreed that haemangioma, particularly the cavernous type, is among the commonest of the orbital tumours, and that its complete removal is usually practicable. Points of note in this case were the early numbness and neuralgic pain around the eye, unaccompanied by any sensory changes, and the evidence of pressure on the optic nerve, reflected in loss of the nasal peripheral visual field, though the optic disc was normal on ophthalmoscopic examination.

- Injury to the nerves supplying the iris, ciliary body, and lateral rectus, was an unfortunate sequel to the operation, though probably this was unavoidable in the mobilisation of a tumour of this size in so confined a space.

\section{OCULAR COMPLICATIONS IN RELAPSINǴ FEVER*}

BY

\author{
MAJOR J. BRUCE HAMILTON \\ A.A.M.C. 2ND A.I.F. ABROAD
}

\section{Introduction}

When Mackenzie in 1830 wrote his "Practical Treatise on Diseases of the Eye," he mentioned that ocular complications occur and should be watched for in relapsing fever. In those prePasteurian days no idea of the aetiology was possible, nor was it anticipated that numerous types of relapsing fever, each due to a different spirochaete, were to be revealed.

Mackenzie's description was of the cosmopolitan louse-borne type of the disease. The delayed ocular complication of amaurosis followed later by acute iritis, as described by him, is typical of the complications I shall enumerate. Also he noted carefully that the iritis recovered long before the amaurosis cleared. He saw in the epidemic in Glasgow 36 cases of ocular complication within 3 months, which is a much greater number than I have examined in the 12 months May 1941 to May 1942.

Since Mackenzie's day several cases of choroiditis accompanying relapsing fever have been reported in the literature; but it is very

* Received October 20, 1942. 